\title{
BTG1 expression in thyroid carcinoma: Diagnostic indicator and prognostic marker
}

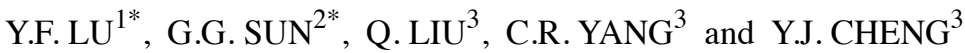 \\ ${ }^{1}$ Department of Endocrinology, Tangshan Workers Hospital; ${ }^{2}$ Department of Chemoradiotherapy, \\ Tangshan People's Hospital, Tangshan 063000; ${ }^{3}$ Department of Radiotherapy, The Fourth Hospital \\ of Hebei Medical University, Shijiazhuang 050017, P.R. China
}

Received March 18, 2014; Accepted May 21, 2014

DOI: $10.3892 /$ ijo.2014.2543

\begin{abstract}
We determined the expression and function of B cell translocation gene 1 (BTG1) in thyroid carcinoma. Thyroid samples were obtained from cancer lesions $(n=83)$ and adjacent normal tissue $(n=35)$ in thyroid cancer patients immediately after endoscopic biopsy. BTG1 expression was determined by immunohistochemistry and western blotting. The effect of BTG1 overexpression was examined in vitro utilizing the human thyroid cancer cell line FTC-133, stably transfected with a recombinant lentivirus (LeBTG1 cells) and compared to empty vector transfected controls (LeEmpty). BTG1 overexpression was verified by reverse transcription quantitative polymerase chain reaction (RT-qPCR) and western blotting. The expression of proteins involved in cell cycle regulation (cyclin D1), apoptosis (Bcl-2) and cell migration (MMP-9) in LeBTG1 cells was analyzed by western blotting. The effect of BTG1 overexpression on cell viability and proliferation was assessed by MTT assay in LeBTG1 and LeEmpty cells. Flow cytometric analyses were used to evaluate the effect of BTG1 expression on cell cycle distribution and apoptosis. The migration and invasion potential of LeBTG1 cells was examined by plating cells in Matrigelcoated chambers. BTG1 protein expression was significantly lower in thyroid cancer tissue biopsies compared to normal tissue as measured by immunohistochemistry (36.1 vs. 80.0\% of tissues; $\mathrm{P}<0.05)$ and western blotting $(0.251 \pm 0.021$ vs.
\end{abstract}

Correspondence to: Dr Yifang Lu, Department of Endocrinology, Tangshan Workers Hospital, no. 27 Wenhua Road, Lubei, Tangshan, Hebei 063000, P.R. China

E-mail: 1yf800218@163.com

Dr Guogui Sun, Department of Chemoradiotherapy, Tangshan People's Hospital, no. 65 Shengli Road, Lunan District, Tangshan, Hebei 063000, P.R. China

E-mail: guogui_sun2013@163.com

*Contributed equally

Key words: B cell translocation gene 1, thyroid carcinoma, cyclin D1, Bcl-2, MMP-9
0.651 $\pm 0.065 ; \mathrm{P}<0.05)$. Decreased expression of BTG1 was significantly correlated with thyroid cancer lymph node metastasis, clinical stage and pathological differentiation $(\mathrm{P}<0.05)$, as well as with reduced overall 10-year survival rates compared to patients with higher expression levels (30.2 vs. $66.7 \%$; $\mathrm{P}<0.05)$. In vitro analyses revealed that LeBTG1 cells had a reduced survival fraction compared to control

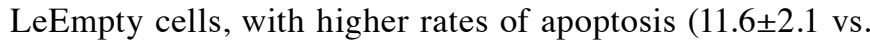
$2.1 \pm 0.4 \%$; $\mathrm{P}<0.05)$. The proportion of LeBTG1 cells in $\mathrm{G} 0 /$ G1 stage and $\mathrm{S}$ phase was also significantly different from LeEmpty cells $(81.8 \pm 6.3$ and $10.2 \pm 1.0 \%$, vs. $62.4 \pm 4.9$ and $25.5 \pm 2.6 \%$, respectively; $\mathrm{P}<0.05$ ), and the migration and invasion of LeBTG1 cells was significantly impaired with respect to LeEmpty cells $(72.0 \pm 8.0$ and $55.0 \pm 7.0$ vs. $113.0 \pm 16.0$ and $89.0 \pm 9.0$, respectively; $\mathrm{P}<0.05)$. These effects were accompanied by decreased protein expression of cyclin D1, Bcl-2 and MMP-9 in LeBTG1 cells $(0.234 \pm 0.018,0.209 \pm 0.021$, $0.155 \pm 0.017$, respectively) compared to control LeEmpty cells $(0.551 \pm 0.065,0.452 \pm 0.043,0.609 \pm 0.072$, respectively; $\mathrm{P}<0.05)$. Reduced BTG1 expression is associated with increased disease severity, suggesting it is a negative regulator of thyroid cancer and can serve as a prognostic indicator.

\section{Introduction}

Cellular proliferation, differentiation, senescence and apoptosis are cell cycle-dependent processes (1). Regulation of the cell cycle is closely linked to tumor development and progression, as it is impaired in almost all tumors $(2,3)$. Moreover, many proto-oncogenes and tumor suppressor genes function as major factors or are directly involved in the regulation of cell cycle (4). Carcinogenic factors can induce mutation, deletion, translocation or amplification of these genes, resulting in de-regulation of the cell cycle, abnormal cell proliferation and tumor development. A new strategy for cancer therapy involves regulating cell cycle, proliferation and apoptosis of tumor cells, and select inhibition of tumor tissue activity (5).

B cell translocation gene 1 (BTG1) is a member of the TOB/BTG family of proteins known to inhibit cell proliferation and negatively regulate the cell cycle that was first identified in B lymphoblastic leukemia (6-9). TOB/BTG family members regulate these processes by acting on cell 
cycle genes in response to various internal and external stimuli. For example, high PC3 expression in NIH/3T3 cells arrests the cell cycle in the G1 phase and inhibits cell growth (10). Enhanced BTG1 expression, which peaks in the G0/G1 phase, promotes the differentiation of neural stem and germ cells and plays an important role in angiogenesis. BTG1 also facilitates the formation of the CCR4/NOT transcriptional complex, which regulates the deadenylation and turnover of cytoplasmic mRNAs. BTG1 is structurally characterized by the presence of a specific BTG domain in the $\mathrm{N}$-terminus along with a large anti-proliferative homologous region (11). Although BTG1 displays some characteristics of tumor suppressor genes $(12,13)$, it is not known if BTG1 is a thyroid cancer suppressor gene. Therefore, this study aimed to determine the roles BTG1 plays in the growth, proliferation, invasion, metastasis and apoptosis of thyroid cancer cells.

\section{Materials and methods}

Reagents. The rabbit anti-human BTG1 monoclonal, rabbit anti-human cyclin D1 polyclonal, rabbit anti-human Bcl-2 polyclonal and rabbit anti-human MMP-9 monoclonal antibodies were purchased from Abcam (Cambridge, UK). Goat anti-rabbit fluorescent secondary antibody (IRDye800) was obtained from LI-COR Bioscience, Inc. (Lincoln, NE, USA). The $\beta$-actin primary antibody, polyoxymethylene and crystal violet were purchased from Sigma (St. Louis, MO, USA). The Plenti6/V5-DEST Vector, lentiviral packaging mix, Opti-MEM, Lipofectamine 2000 and TRIzol were obtained from Invitrogen (Thermo Fisher Scientific, Waltham, MA, USA). An immunohistochemistry kit and the Annexin V-FITC/PI apoptosis detection kit were purchased from 4A Biotech Co., Ltd. (Beijing, China). Fetal bovine serum (FBS), cell-culture media and supplementary materials were obtained from Gibco (Thermo Fisher Scientific, Waltham, MA, USA). Reverse transcriptase reagents and SYBR Premix ExTaq (Perfect Real-Time) were purchased from Takara (Otsu, Shiga, Japan). Invasion chambers and Matrigel for invasion and migration assays were purchased from BD Biosciences (Franklin Lakes, NJ, USA).

Clinical data. All patients enrolled in this study provided informed consent in advance. There were 23 males and 60 females, aged from 26 to 73 years old, with a median age of 44 years. The studies contained 46 cases of papillary cancer, 22 cases of follicular cancer, and 11 cases of medullary cancer, and 4 cases of undifferentiated cancer. Of the 83 cases of thyroid cancer, 31 had lesions $\leq 4 \mathrm{~cm}$ and 52 had esions $>4 \mathrm{~cm}$. Fifty-five of the patients demonstrated lymph node metastasis. Twenty-seven patients had grade I (well differentiated) tumors, and 56 patients had grade II or III (moderately to poorly differentiated) tumors. Clinical staging showed 25 cases of stage I-II, and 58 cases of stage III-IV. Two endoscopic biopsy samples were collected from each patient and either immediately stored in liquid nitrogen for western blot analysis, or fixed in a $4 \%$ formaldehyde solution and paraffin-embedded for immunohistochemistry.

Cell culture and gene transfection. The human thyroid cancer cell line FTC-133 was maintained in DMEM medium supplemented with $10 \%$ FBS, which was changed every 2-3 days. Upon reaching confluence, cells were subcultured with $0.25 \%$ trypsin and $1 \%$ ethylenediaminetetraacetic acid. BTG1 cDNA sequences were cloned into the BamHI and AscI sites of the plenti6/V5-DEST vector, and cells were transfected with pLenti6-BTG1 or plenti6/V5-DEST vector using Lipofectamine 2000. Transfected cells were maintained in blastidicin (5 $\mu \mathrm{g} / \mathrm{ml})$-containing RPMI-1640 medium for selection of stable vector-containing sublines.

Immunohistochemistry. Immunohistochemistry was performed as previously described (14). Briefly, 4- $\mu \mathrm{m}$ sections were prepared from paraffin-embedded biopsy samples and dehydrated. Sections were incubated in $3 \%$ hydrogen peroxide for $10 \mathrm{~min}$ to block endogenous peroxidase, followed by $20 \mathrm{~min}$ in $0.05 \%$ trypsin. Sections were incubated for $20 \mathrm{~min}$ at room temperature in a blocking solution containing $10 \%$ goat serum followed by BTG1 antibody $(1: 100)$ at $4{ }^{\circ} \mathrm{C}$ overnight. For a negative control, the primary antibody was replaced with phosphate-buffered saline (PBS). Sections were subsequently incubated for $20 \mathrm{~min}$ each in secondary and tertiary antibodies at room temperature, visualized by DAB staining and countered with a hematoxylin stain. Two pathologists blind to patient condition examined and quantified the sections. Five randomly selected fields from three slides for each specimen were examined under a microscope and counted. BTG1 expression was determined based on the percentage of positive cells ( 0 points, $\leq 5 \%$; 1 point, $5-25 \%$; 2 points, $25-50 \%$; and 3 points, $>50 \%$ positive cells) and the staining intensity [0 points, no staining; 1 point, weak staining (light yellow); 2 points, moderate staining (yellowish-brown); and 3 points, strong staining (brown)]. The final score of BTG1 expression was the product of the BTG1 expression rate (percentage score) and intensity: - for 0 points, + to +++ for positive (+ for 1-3 points, ++ for $4-6$ points and +++ for 7-9 points).

Quantitative real-time $R T-P C R$. Total RNA was extracted from FTC-133 cells using TRIzol reagent according to the manufacturer's protocol. Total RNA (500 ng) was reverse transcribed using reverse transcriptase, and quantitative real-time RT-PCR (qRT-PCR) was performed on an ABI PRISM 7300 Real-Time PCR system (Applied Biosystems, Inc., by Life Technologies/Thermo Fisher Scientific, Waltham, MA, USA) according to the standard manufacturer's protocol for SYBR Premix ExTaq. Gene specific primers used include: BTG1, sense 5'-GGAATTCATGCATCCCTTCTACACCCGG and antisense 5'-CGACGCGTTTAACCTGATACAGTCATCAT; and $\beta$-actin for normalization, sense 5'-ATCGTCCACC GCAAATGCTTCTA and antisense 5'-AGCCATGCCAA TCTCATCTTGTT. Thermal cycling conditions were $95^{\circ} \mathrm{C}$ for $1 \mathrm{~min}$, followed by 40 cycles of $95^{\circ} \mathrm{C}$ for $15 \mathrm{sec}$ and $60^{\circ} \mathrm{C}$ for $1 \mathrm{~min}$. The expression level relative to $\beta$-actin was calculated using the $2^{-\Delta \Delta C t}$ method in SDS 1.3 software.

Western blot analysis. Western blotting was performed as previously described (15). Briefly, $50 \mu \mathrm{g}$ of protein (determined using a BCA Protein Assay kit (Tiangen Biotech Co., Ltd., Beijing, China) per samples were subjected to sodium dodecyl sulfate-polyacrylamide gel electrophoresis and transferred to 
Table I. Expressions of BTG1 in normal and cancerous thyroid tissue.

Expression of BTG1 protein

\begin{tabular}{lcrrrrrr}
\cline { 3 - 7 } Group & Case & - & + & ++ & +++ & $\chi^{2}$ & P-value \\
\hline Normal tissue & 35 & 7 & 6 & 13 & 9 & 22.721 & 0.000 \\
Cancer tissue & 83 & 53 & 13 & 10 & 7 & & \\
\hline
\end{tabular}

a nitrocellulose membrane. Membranes were incubated for $2 \mathrm{~h}$ in $5 \%$ non-fat dry milk followed by an overnight incubation at $4^{\circ} \mathrm{C}$ in primary antibody (BTG1, 1:1000; $\beta$-actin, 1:5000). After washing, the membranes were incubated with goat antirabbit fluorescent secondary antibody (IRDye800, 1:20,000 dilution) in the dark, for $1 \mathrm{~h}$, at room temperature. The blots were then scanned and analyzed using the Odyssey Infrared Imaging System (LI-COR Bioscience, Inc.). Western blot data were quantified by normalizing the BTG1 signal intensity of each sample to that of $\beta$-actin.

MTT assay. Cell viability was determined using the tetrazolium salt MTT (3-[4,5-dimethylthiazol-2-yl]-2,5-diphenyltetrazolium bromide) assay, as previously described (16). Briefly, FTC-133 cells were plated into 96-well culture plates at an optimal density of $5 \times 10^{3}$ cells $/ \mathrm{ml}$ with $200 \mu \mathrm{l} /$ well. After 24-96 h, $20 \mu \mathrm{l}$ of $5 \mathrm{mg} / \mathrm{ml}$ MTT was added to each well and incubated at $37^{\circ} \mathrm{C}$ for $4 \mathrm{~h}$. The medium was then gently aspirated and $150 \mu \mathrm{l}$ of dimethyl sulfoxide was added to each well to solubilize the formazan crystals. The optical density of each sample was immediately measured at $570 \mathrm{~nm}$ using a microplate reader (Bio-Rad, Hercules, CA, USA).

Flow cytometry. An Annexin V-FITC flow cytometry assay was used as previously described (17) to detect the apoptosis rate. Cells were plated into $60-\mathrm{mm}$ dishes for $48 \mathrm{~h}$ and grown to $70-75 \%$ confluency. Cells were then collected, washed with ice-cold PBS, and resuspended at a density of $1 \times 10^{6}$ cells $/ \mathrm{ml}$ in a binding buffer and incubated for $15 \mathrm{~min}$ in the dark at $25^{\circ} \mathrm{C}$ with $5 \mu \mathrm{l}$ of Annexin V-FITC and $10 \mu \mathrm{l}$ of propidium iodide $(20 \mu \mathrm{g} / \mathrm{ml})$. Ten thousand cells were analyzed with a FACScan flow cytometer with CellQuest software (BD Biosciences) for apoptosis rate determination. For cell cycle distribution, $1 \times 10^{6}$ cells were fixed in $70 \%$ ethanol and resuspended in $1 \mathrm{ml}$ of a solution containing $3.8 \mathrm{mM}$ sodium citrate, $50 \mu \mathrm{g} / \mathrm{ml}$ propidium iodide, and $0.5 \mu \mathrm{g}$ of RNase A, and analyzed with the flow cytometer using the ModFit software program (Verity Software House, Topsham, ME, USA).

Invasion and migration assays. Invasion and migration assays were performed as previously described (18). Briefly, $10 \times 10^{5}$ cells were plated into Invasion Chambers with Costar Transwell $8 \mu \mathrm{m}$ inserts coated with $50 \mu \mathrm{g}$ reduced serum Matrigel according to the manufacturer's instructions. Medium supplemented with $10 \%$ FBS was used in the lower chamber. Migration assays were performed in the same manner excluding the Matrigel. After $12 \mathrm{~h}$, non-invading cells and media were removed with a cotton swab. Cells on the lower surface of the membrane were fixed with polyoxymethylene and stained with $0.1 \%$ crystal violet for $30 \mathrm{~min}$. Stained cells were counted under a microscope in four randomly selected fields, and the average was used to indicate cell migration and invasion.

Statistical analyses. All statistical analyses were performed using SPSS 16.0 software (IBM, Armonk, NY, USA), according to published guidelines (19). Survival distributions were estimated with the Kaplan-Meier method and compared with the log-rank test. Student's t-test, $\chi^{2}$ and Fisher's exact tests were used to analyze the differences between groups. Data are presented as the mean \pm the standard error, and a $\mathrm{P}<0.05$ was considered to be statistically significant.

\section{Results}

BTG1 protein expression in normal tissue and thyroid cancer. Immunohistochemistry for BTG1 revealed light yellow to brown staining in $80.0 \%$ (28/35) of normal thyroid tissues, and negative or weak staining in $36.1 \%$ (30/83) thyroid cancer tissues $(\mathrm{P}<0.05)$ (Table I, Fig. 1). Furthermore, BTG1 protein expression was significantly lower in cancer lesion samples compared to adjacent normal tissue, as determined by western blot analysis $(0.251 \pm 0.021$ vs. $0.651 \pm 0.065 ; \mathrm{P}<0.05)$ (Fig. 2). BTG1 expression levels correlated with lymph node metastasis, clinical stage and pathological differentiation $(\mathrm{P}<0.05)$, regardless of age, gender, tumor size and pathological types (P>0.05) (Table II).

BTG1 expression and prognosis. Follow-up examinations were performed on cancer patients for up to 120 months, with 36 patients remaining at the conclusion of the study. Overall survival (OS) rates were determined between patients positive for BTG1 expression and those negative for expression. Sixteen of the 53 individuals showing no BTG1 expression remained at the conclusion of the study, with an OS rate of $30.2 \%$. Patients positive for BTG1 expression had a significantly higher OS rate of $66.7 \%(20 / 30)(\mathrm{P}<0.05)$ (Fig. 3).

Stable transfection of BTG1 in thyroid cancer cells. BTG1 overexpressing FTC-133 cells (named LeBTG1) were obtained by a stable transfection of BTG1 cDNA, and compared with FTC-133 cells overexpressing an empty vector (named LeEmpty) as a control. Analysis of qRT-PCR data showed LeBTG1 cells had a significantly higher expression of 


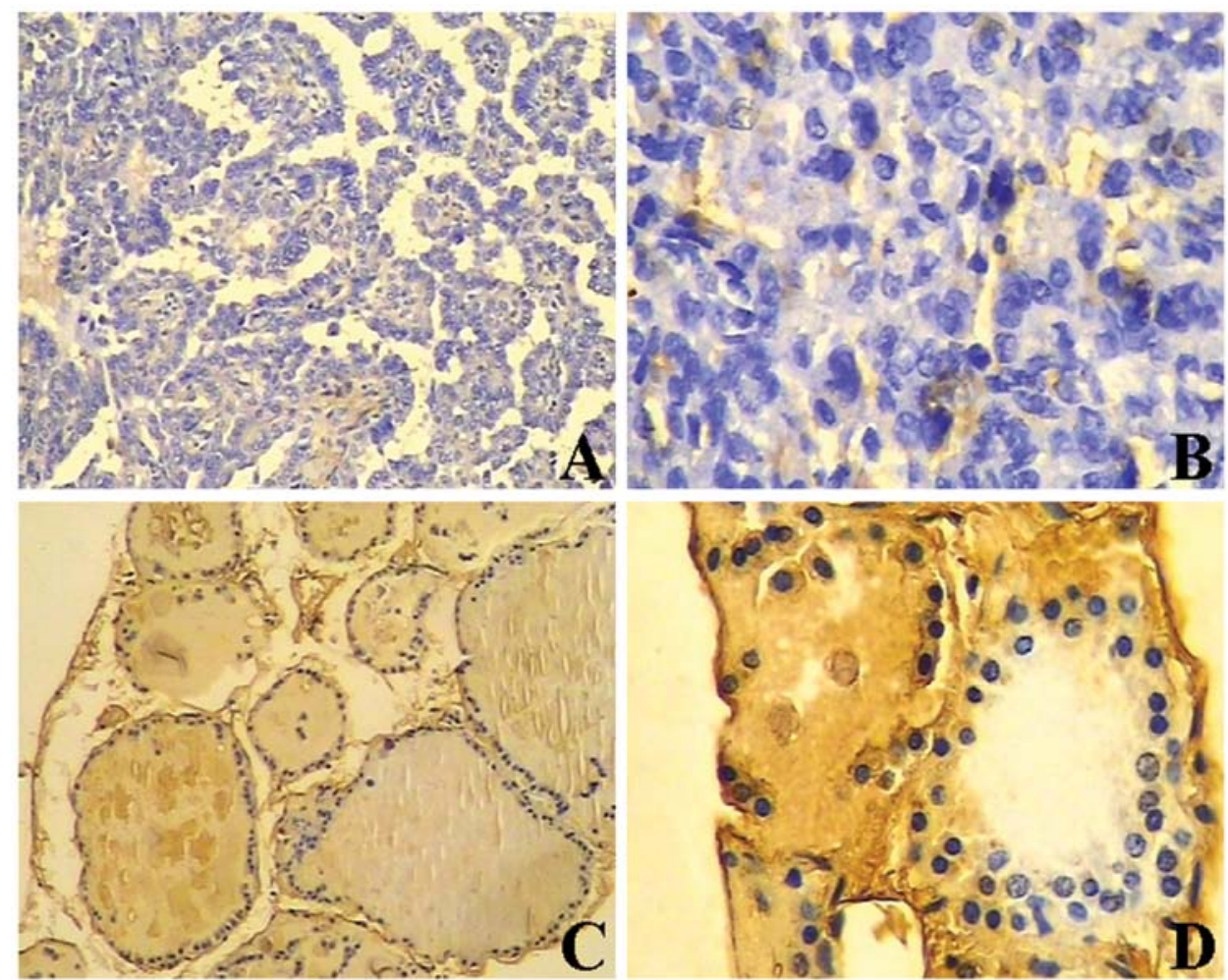

Figure 1. BTG1 immunohistochemistry. BTG1 staining in thyroid carcinoma (A, magnification x100; B, magnification x400); BTG1 staining in normal tissue (C, magnification x100; D, magnification x400).
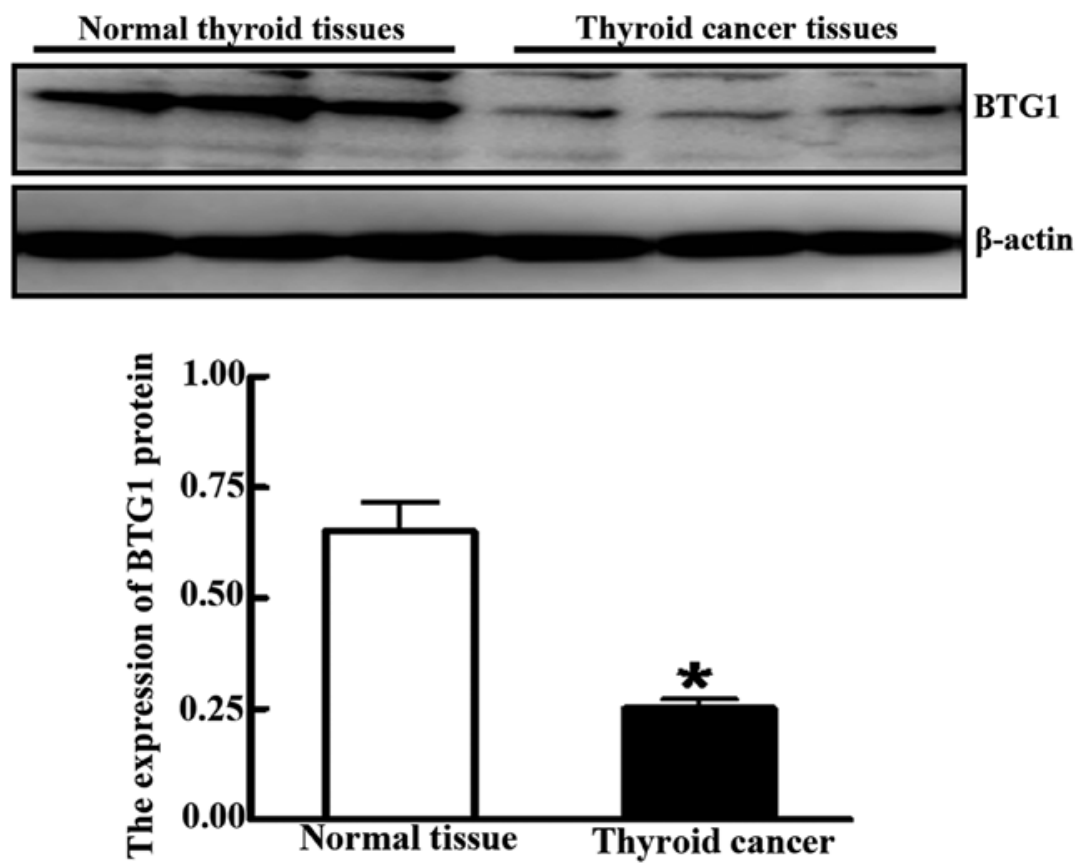

Figure 2. BTG1 protein levels. BTG1 protein levels were assessed by western blot analysis (upper panel). Quantification (lower panel) revealed that protein levels were significantly reduced relative to $\beta$-actin in cancer tissues compared to normal tissues; ${ }^{*} \mathrm{P}<0.05$.

BTG1 mRNA compared to LeEmpty cells $(0.912 \pm 0.097$ vs. $0.423 \pm 0.042 ; \mathrm{P}<0.05$ ) (Fig. 4A). Furthermore, western blot analysis showed that LeBTG1 cells had a significantly higher expression of BTG1 protein compared to LeEmpty cells ( $0.873 \pm 0.086$ vs. $0.395 \pm 0.042$; $\mathrm{P}<0.05)$ (Fig. 4B).
Cellular effects of BTG1 overexpression. LeBTG1 cells had a significantly lower viability at $24,48,72$ and $96 \mathrm{~h}$ compared to LeEmpty cells as assessed by the MTT assay ( $\mathrm{P}<0.05)$ (Fig. 5). Cell cycle analysis using flow cytometry showed that the proportion of LeBTG1 cells in G0/G1 and S phases of the cell cycle 


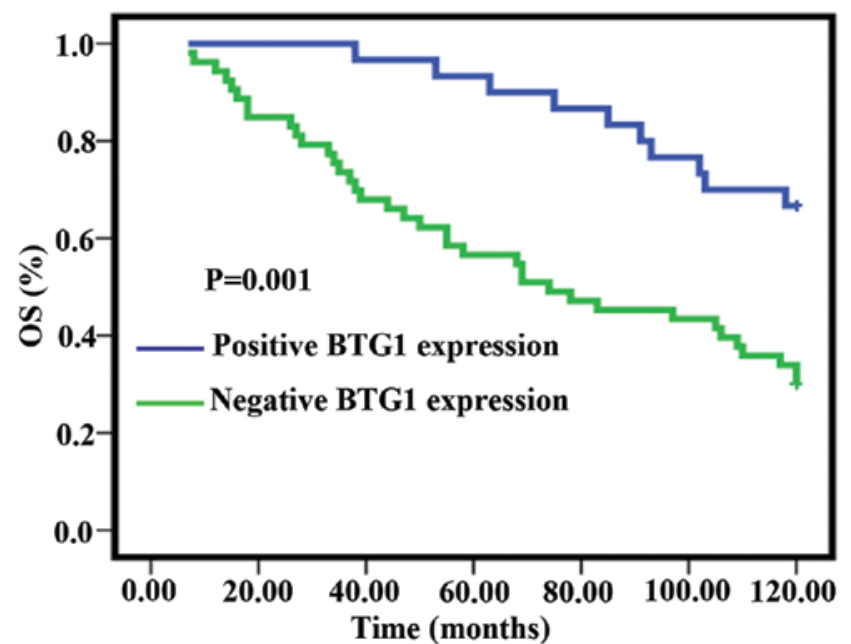

Figure 3. Overall survival in thyroid cancer patients. Thyroid cancer patients positive for BTG1 expression has a significantly higher survival rate compared to those negative for this expression; $\mathrm{P}<0.05$.

Table II. The relationship between BTG1 expression and thyroid cancer characteristics.

\begin{tabular}{lrrrrr} 
& & \multicolumn{4}{c}{ Expression of BTG1 protein } \\
\cline { 3 - 6 } Group & Case & - & + to +++ & $\chi^{2}$ & P-value \\
\hline Gender & 23 & 15 & 8 & 0.026 & 0.873 \\
Male & 60 & 38 & 22 & & \\
Female & & & &
\end{tabular}

Age (years)

$\begin{array}{rrrrrr}\leq 40 & 63 & 40 & 23 & 0.015 & 0.903 \\ >40 & 20 & 13 & 7 & & \end{array}$

Pathological types

Papillary cancer

$\begin{array}{rrrr}46 & 29 & 17 & 0.845 \\ 22 & 13 & 9 & \\ 11 & 8 & 3 & \\ 4 & 3 & 1 & \end{array}$

0.839

Follicular cancer

Medullary cancer

Undifferentiated

cancer

Tumor length $(\mathrm{cm})$

$\begin{array}{llllll}\leq 4 & 31 & 18 & 13 & 0.719 & 0.396 \\ >4 & 52 & 35 & 17 & & \end{array}$

Lymph node

metastasis

$\begin{array}{llllll}\mathrm{N} 0 & 28 & 12 & 16 & 8.072 & 0.004 \\ \mathrm{~N}+ & 55 & 41 & 14 & & \end{array}$

Clinical stages

$\begin{array}{llllll}\text { I-II } & 25 & 10 & 15 & 8.821 & 0.003 \\ \text { III-IV } & 58 & 43 & 15 & & \end{array}$

Histological grade

\begin{tabular}{llllll} 
I & 27 & 11 & 16 & 9.264 & 0.002 \\
II-III & 56 & 42 & 14 & & \\
\hline
\end{tabular}
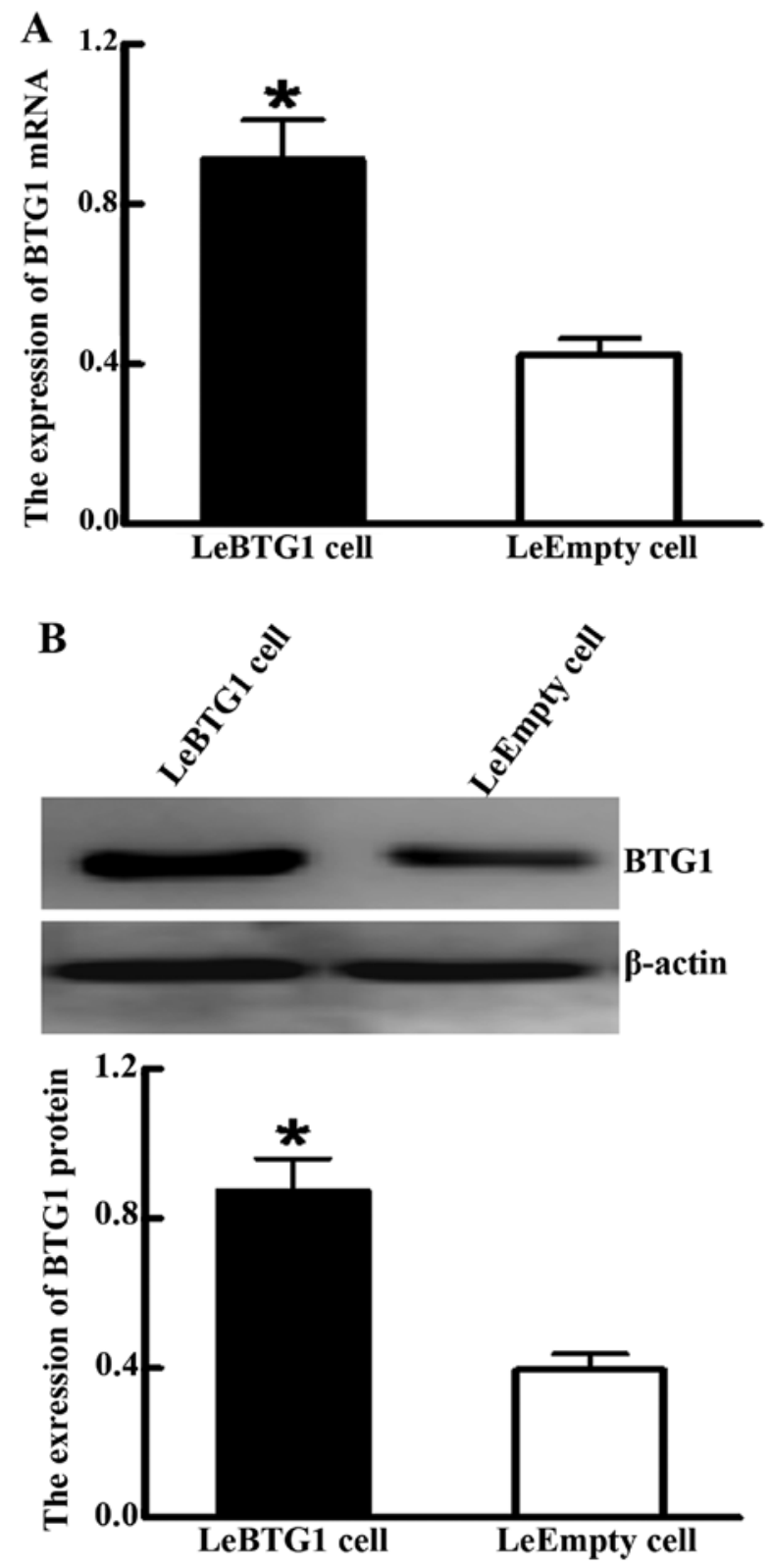

Figure 4. Expression of BTG1 in a stable transfected cell line. (A) LeBTG1 cells express significantly more BTG1 mRNA as determined by quantitative RT-PCR; (B) Western blot analysis revealed that LeBTG1 cells express more BTG1 protein; levels relative to $\beta$-actin, ${ }^{*} \mathrm{P}<0.05$ compared to LeEmpty cells.

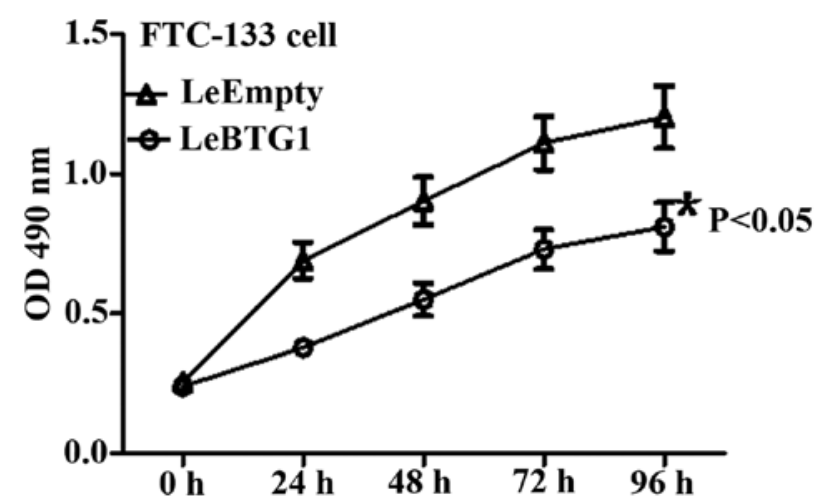

Figure 5. Reduced cell viability with BTG1 overexpression. An MTT assay demonstrated that LeBTG1 cells had significantly reduced activity levels at $24,48,72$ and $96 \mathrm{~h}$, indicating reduced viability and slower growth $(\mathrm{P}<0.05)$. 

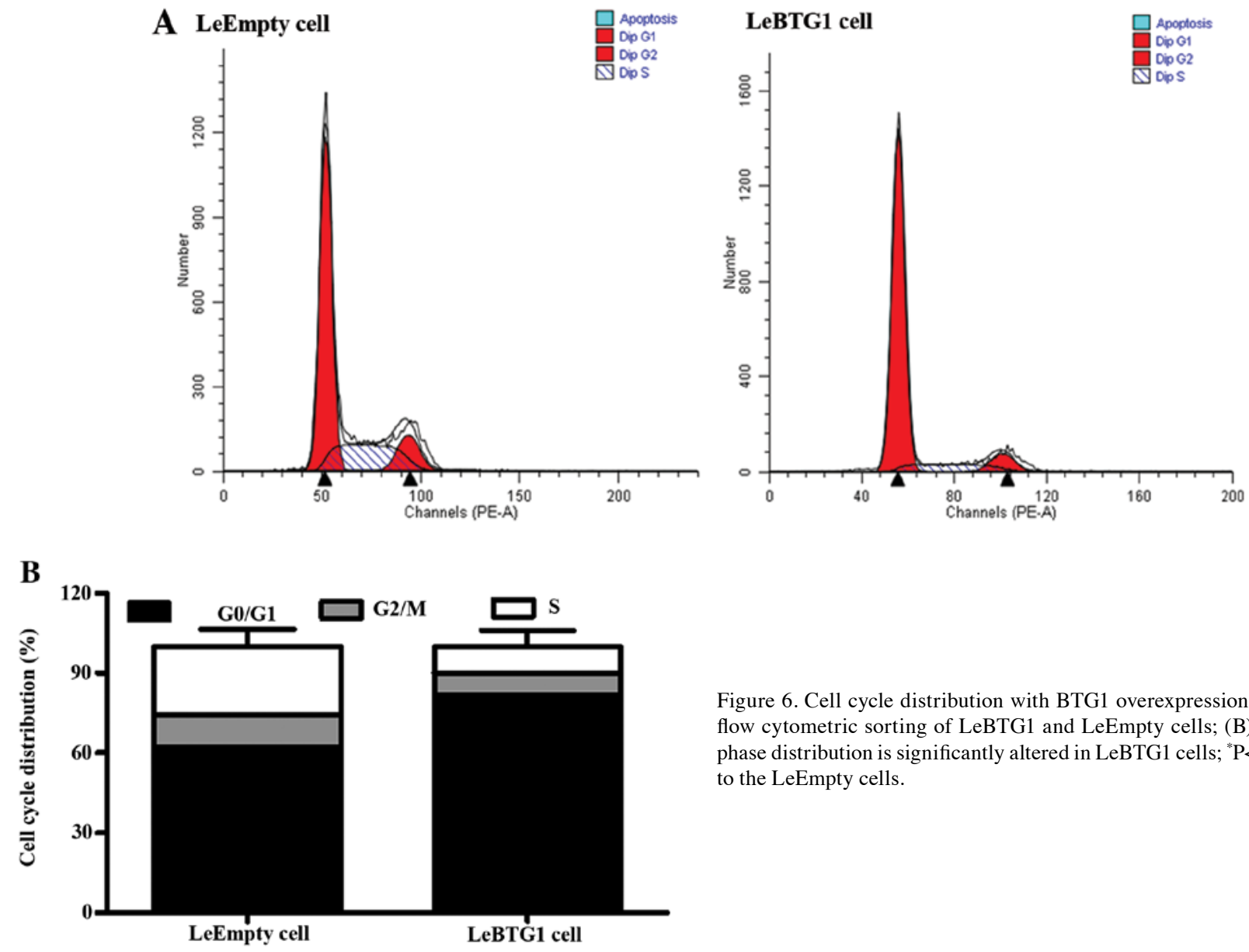

Figure 6. Cell cycle distribution with BTG1 overexpression. (A) Results of flow cytometric sorting of LeBTG1 and LeEmpty cells; (B) The cell cycle phase distribution is significantly altered in LeBTG1 cells; ${ }^{*} \mathrm{P}<0.05$ compared to the LeEmpty cells.
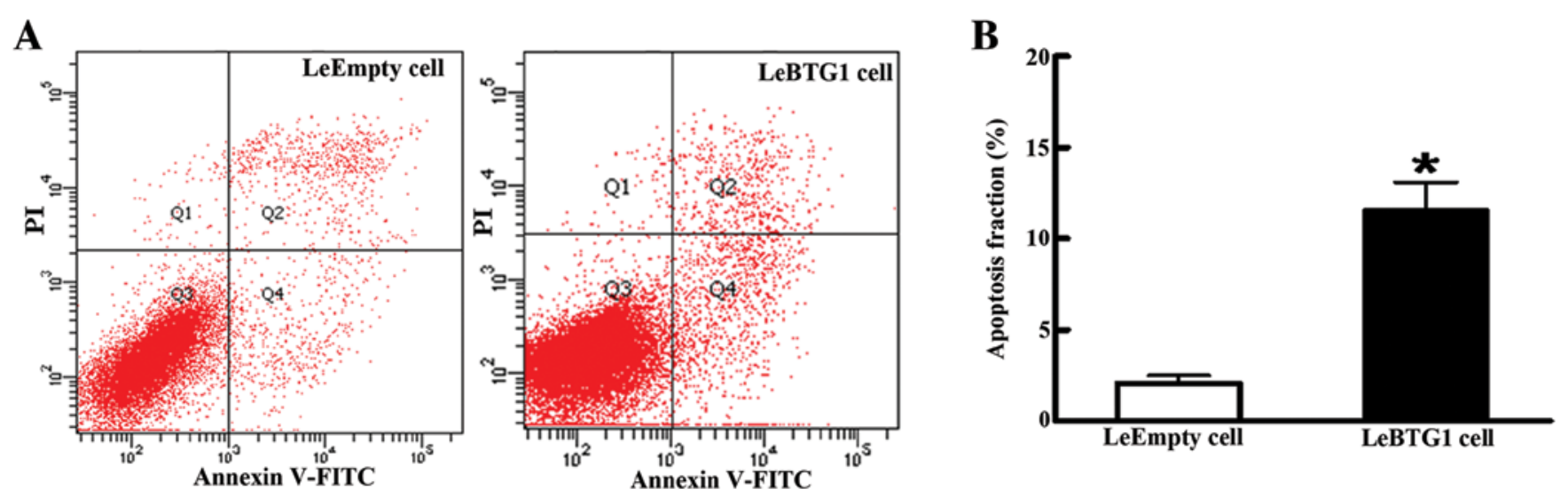

Figure 7. Increased apoptosis with BTG1 overexpression. (A) Results of flow cytometric sorting of LeBTG1 and LeEmpty cells; (B) A higher percentage of LeBTG1 cells were apoptotic compared to LeEmpty cells; ${ }^{*} \mathrm{P}<0.05$.

were significantly different compared to the control LeEmpty cells $(81.8 \pm 6.3$ and $10.2 \pm 1.0 \%$, vs. $62.4 \pm 4.9$ and $25.5 \pm 2.6 \%$, respectively; $\mathrm{P}<0.05$ ) (Fig. 6). In addition, there was a large increase in the early apoptosis rate in LeBTG1 cells compared to control LeEmpty cells $(11.6 \pm 2.1$ vs. $2.1 \pm 0.4 \%$; $\mathrm{P}<0.05)$ (Fig. 7). Furthermore, LeBTG1 cells had a reduced capability for invasion and migration through Transwell inserts (72.0 \pm 8.0 and 55.0 \pm 7.0 , respectively) compared to control LeEmpty cells $(113.0 \pm 16.0$ and 89.0 \pm 9.0 , respectively; $\mathrm{P}<0.05)$ (Fig. 8).
To further identify the mechanisms by which BTG1 overexpression regulated these cellular changes in cancer cells, expression levels of proteins critical for the regulation of cell cycle, apoptosis and migration, were examined. Western blot analysis revealed that LeBTG1 cells had significantly reduced levels of cyclin D1, Bcl-2 and MMP-9 (0.234 \pm 0.018 , $0.209 \pm 0.021$ and $0.155 \pm 0.017$, respectively) compared to control LeEmpty cells $(0.551 \pm 0.065,0.452 \pm 0.043$ and $0.609 \pm 0.072$, respectively; $\mathrm{P}<0.05$ ) (Fig. 9). 
A
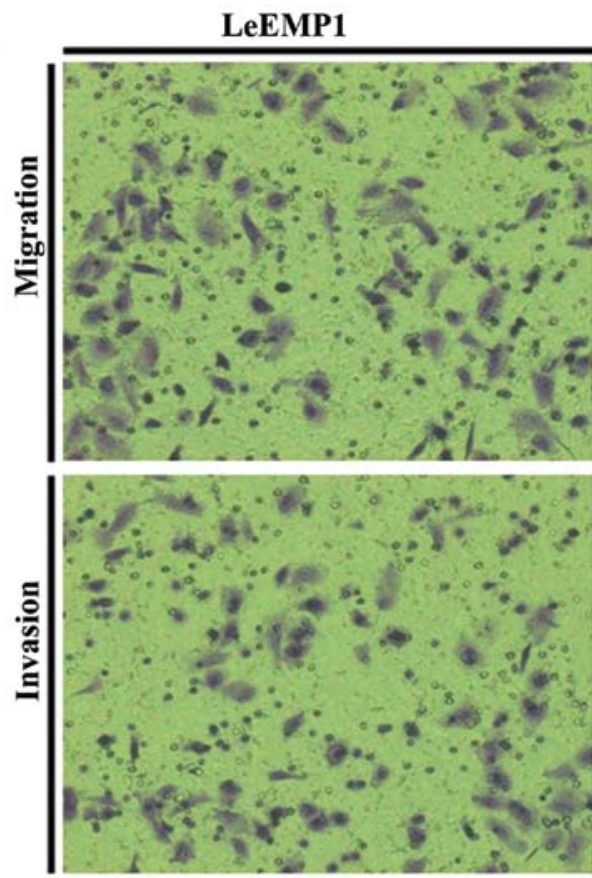

LeEmpty
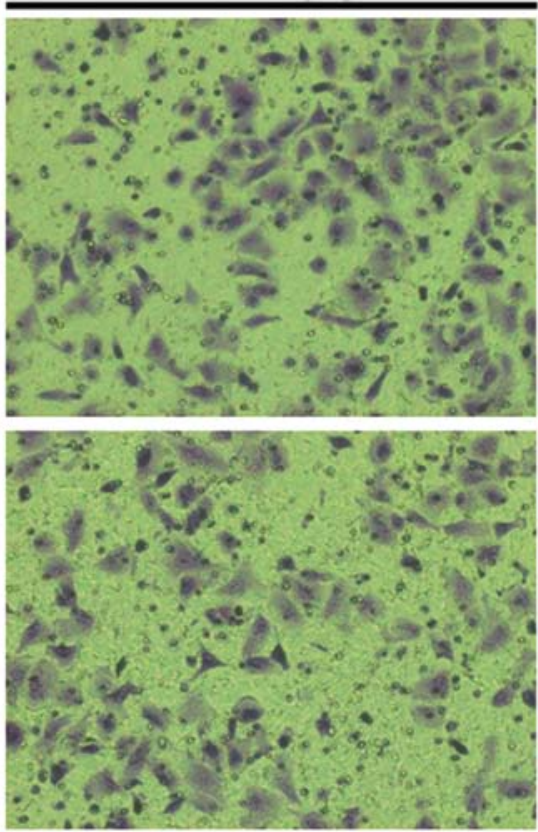
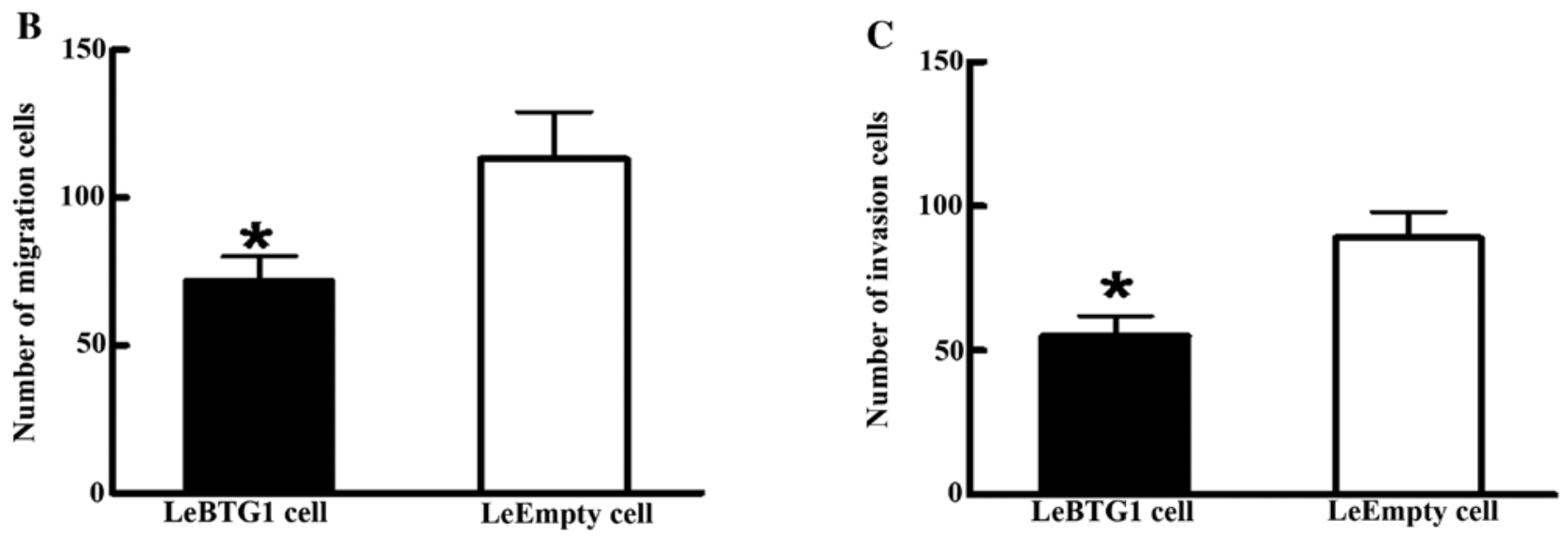

Figure 8. Reduced cell migration and invasion with BTG1 overexpression. (A) Photomicrographs showing cellular invasion and migration of LeBTG1 and LeEmpty cells through Transwell $8 \mu \mathrm{m}$ inserts (magnification, $\mathrm{x} 400$ ); (B) Quantification of migrating cells; (C) Quantification of invading cells; ${ }^{*} \mathrm{P}<0.05$ compared to control LeEmpty cells.

\section{Discussion}

Tumor development and progression are associated with uncontrolled proliferation and reduced apoptosis of tumor cells. BTG1 has been show to act as a tumor suppressor gene in breast cancer, by inhibiting proliferation, regulating cell cycle and inducing apoptosis (20). This study examined BTG1 protein expression in thyroid cancer tissue and showed that levels were significantly lower and were correlated with lymph node metastasis, clinical stage and cancer differentiation. Current studies suggest that tumor stage is the preferred prognostic indicator (21), though prognoses can still vary considerably among patients in the same stage. Therefore, it is of particular importance to identify reliable molecular markers for use in clinical practice. The results of this study suggest that BTG1 deletion is a major contributor to the development and progression of thyroid cancer. As expression-positive patients had a significantly higher 10 -year overall survival rate, BTG1 may be a useful prognostic indicator for patients with thyroid cancer. The combination of the tumor-node-metastasis classification system and BTG1 expression scores may provide some valuable information for clinicians in choosing treatment options, and for predicting disease severity and prognosis.

The development of thyroid cancer is driven by the abnormal proliferation of cells that normally undergo apoptosis (22). Recent studies (20) have indicated that overexpression of BTG1 can affect the cell cycle and suppress tumor growth. This study utilized in vitro tests to confirm that thyroid cancer cells with high BTG1 expression had significantly weakened viability and proliferation potential. In addition, BTG1 overexpression resulted in decreased protein levels of cyclin D1, which is considered to be a proto-oncogene product that is highly expressed or mutated in a variety of human tumors (23). The increased BTG1 expression in thyroid cancer FTC-133 cells resulted in a higher proportion of cells in the G0/G1 phase, suggesting the occurrence of G0/G1 arrest and inhibi- 


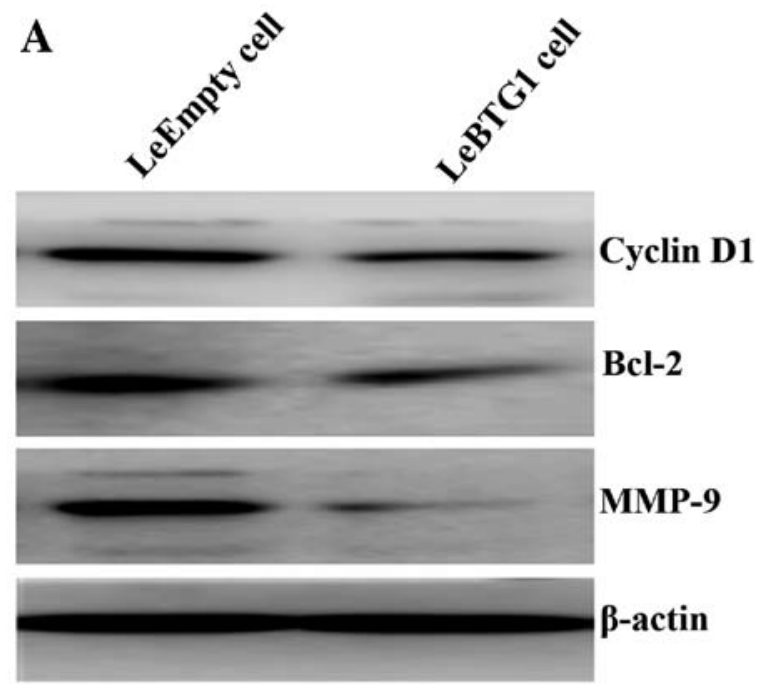

\section{B G}

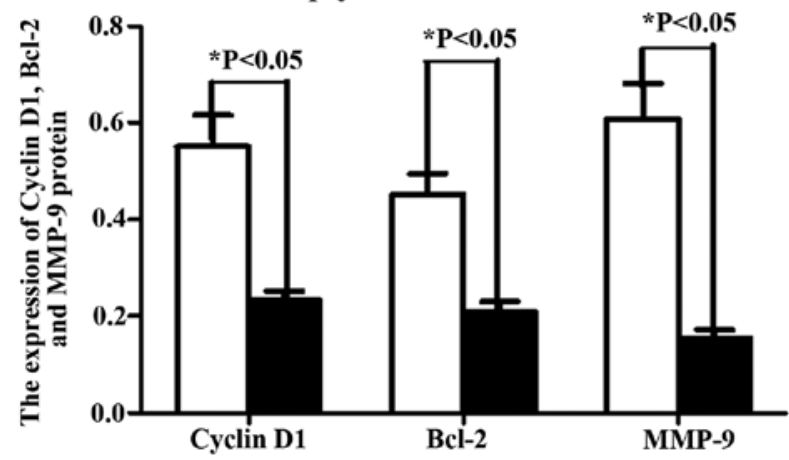

Figure 9. Reduced protein levels of cyclin D1, Bcl-2 and MMP-9 with BTG1 overexpression. (A) Representative western blots showing protein expression in LeBTG1 and LeEmpty cells; (B) Quantification of cyclin D1, Bcl-2 and MMP-9 expression relative to $\beta$-actin; ${ }^{*} \mathrm{P}<0.05$ compared to LeEmpty cells.

tion of growth. Taken together, the data implicate BTG1 in cell cycle regulation and cyclin D1 expression.

In this study, increased BTG1 expression in thyroid cancer cells reduced the amount of the anti-apoptotic protein Bcl-2 and induced apoptosis. Apoptosis is a programmed death process that involves a series of changes in relevant genes, including $\mathrm{Bcl}-2$ and caspase family genes, oncogenes such as C-myc, and the tumor suppressor gene p53 (24), and is regulated by numerous internal and external factors (25). These results are in agreement with previous work linking BTG1 expression with apopotosis. Corjay et al demonstrated a high level of BTG1 expression in apoptotic cells within macrophage-rich tissues in patients with hereditary hyperlipidemia (26), and Lee et al showed that BTG1 could induce apoptosis in glioma cells (27). Moreover, a study by Nahta et al found that apoptosis in breast cancer MCF7 cells induced by knockdown of Bcl-2 was regulated by BTG1 expression (28). Therefore, the evidence suggests that BTG1 can inhibit the growth of thyroid cancer cells by reducing Bcl-2 expression.

Tumor invasion and metastasis are major causes for treatment failures, thus the ultimate research goals are to identify the molecular mechanisms underlying metastasis and target key pathways that inhibit this process. Tumor invasion and metastasis share common molecular mechanisms and involve a number of changes in tumor cells and the microenvironment, including altered tumor cell adhesion properties, enhanced proliferation, survival, chemotaxis and migration of tumor cells, lymphangiogenesis, evasion of immune attack, and hydrolysis of surrounding matrix proteins (29). A key step in tumor invasion and clonal growth is the remodeling of the extracellular matrix and basement membranes through proteolytic degradation by MMPs, which are highly expressed by tumor cells with malignant, invasive and metastatic phenotypes. Moreover, the degree of malignancy and patient prognosis are associated with excessive expression of MMP-2 and MMP-9 (30,31). Tumor cells can also regulate the expression of MMPs produced by stromal cells by secreting chemokines, cytokines and extracellular MMP inducer, a cell surface glycoprotein. This study showed that BTG1-overexpressing thyroid cancer cells had decreased MMP-9 protein levels and reduced invasion and migration in vitro, suggesting that BTG1 could modulate tumor cell metastasis by downregulating MMP-9 expression.

In conclusion, this study provides clinical and in vitro evidence implicating BTG1 in the development and progression of thyroid cancer. The results show that BTG1 protein levels were significantly reduced in thyroid cancer biopsy specimens and were associated with disease progression and prognosis. Furthermore, the effects of BTG1 expression on the regulation of cancer cell proliferation, apoptosis, invasion and metastasis suggest that BTG1 expression may serve as a prognostic marker for thyroid cancer patients.

\section{References}

1. Agathocleous M and Harris WA: Metabolism in physiological cell proliferation and differentiation. Trends Cell Biol 23: 484-492, 2013.

2. Hofmockel G: Molecular genetic principles of tumor development and progression. Urologe A 39: 212-213, 2000 (In German).

3. Shibata D and Aaltonen LA: Genetic predisposition and somatic diversification in tumor development and progression. Adv Cancer Res 80: 83-114, 2001.

4. Lee EY and Muller WJ: Oncogenes and tumor suppressor genes. Cold Spring Harb Perspect Biol 2: a003236, 2010.

5. Okuyama T, Maehara Y, Kabashima A, Takahashi I, Kakeji Y and Sugimachi K: Combined evaluation of expressions of p53 and $\mathrm{p} 21$ proteins as prognostic factors for patients with gastric carcinoma. Oncology 63: 353-361, 2002.

6. Vadgama JV, Scuric Z, Chakrabarti R, Marzo E, Shen D and Wu Y: Insulin-like growth factor I differentially regulates the expression of HIRF1/hCAF1 and BTG1 genes in human MCF-7 breast cancer cells. Int J Mol Med 18: 129-139, 2006.

7. Cortes U, Moyret-Lalle C, Falette N, Duriez C, Ghissassi FE, Barnas C, Morel AP, Hainaut P, Magaud JP and Puisieux A: BTG gene expression in the p53-dependent and -independent cellular response to DNA damage. Mol Carcinog 27: 57-64, 2000.

8. Winkler GS: The mammalian anti-proliferative BTG/Tob protein family. J Cell Physiol 222: 66-72, 2010.

9. Rouault JP, Rimokh R, Tessa C, Paranhos G, Ffrench M, Duret L, Garoccio M, Germain D, Samarut J and Magaud JP: BTG1, a member of a new family of antiproliferative genes. EMBO J 11: 1663-1670, 1992.

10. Rouault JP, Falette N, Guéhenneux F, Guillot C, Rimokh R, Wang Q, Berthet C, Moyret-Lalle C, Savatier P, Pain B, Shaw P, Berger R, Samarut J, Magaud JP,Ozturk M, Samarut C and Puisieux A: Identification of BTG2, an antiproliferative p53-dependent component of the DNA damage cellular response pathway. Nat Genet 14: 482-486, 1996.

11. Matsuda S, Rouault J, Magaud J and Berthet C: In search of a function for the TIS21/PC3/BTG1/TOB family. FEBS Lett 497: 67-72, 2001. 
12. Bozec A, Peyrade F and Milano G: Molecular targeted therapies in the management of head and neck squamous cell carcinoma: recent developments and perspectives. Anticancer Agents Med Chem 13: 389-402, 2013.

13. Suzuki K, Nakamura K, Kato K, Hamada $\mathrm{H}$ and Tsukamoto $\mathrm{T}$ : Exploration of target molecules for prostate cancer gene therapy. Prostate 67: 1163-1173, 2007.

14. Turashvili G, Bouchal J, Ehrmann J, Fridman E, Skarda J and Kolar Z: Novel immunohistochemical markers for the differentiation of lobular and ductal invasive breast carcinomas. Biomed Pap Med Fac Univ Palacky Olomouc Czech Repub 151: 59-64, 2007.

15. Ranganathan V and De PK: Western blot of proteins from Coomassie-stained polyacrylamide gels. Anal Biochem 234: 102-104, 1996

16. van Meerloo J, Kaspers GJ and Cloos J: Cell sensitivity assays: the MTT assay. Methods Mol Biol 731: 237-245, 2011.

17. Rasola A and Geuna M: A flow cytometry assay simultaneously detects independent apoptotic parameters. Cytometry 45: 151-157, 2001.

18. Kramer N, Walzl A, Unger C, Rosner M, Krupitza G Hengstschläger $\mathrm{M}$ and Dolznig $\mathrm{H}$ : In vitro cell migration and invasion assays. Mutat Res 752: 10-24, 2013.

19. Richards RJ: Responsibility for statistical analyses. Endocr Pract 9: 329,2003

20. Zhu R, Zou ST, Wan JM, Li W, Li XL and Zhu W: BTG1 inhibits breast cancer cell growth through induction of cell cycle arrest and apoptosis. Oncol Rep 30: 2137-2144, 2013.

21. Manjili MH, Najarian K and Wang XY: Signatures of tumorimmune interactions as biomarkers for breast cancer prognosis Future Oncol 8: 703-711, 2012.

22. Martinez-Outschoorn UE, Pavlides S, Sotgia F and Lisanti MP: Mitochondrial biogenesis drives tumor cell proliferation. Am J Pathol 178: 1949-1952, 2011.
23. Koff A, Cross F, Fisher A, Schumacher J,Leguellec K, Philippe M and Roberts JM: Human cyclin E, a new cyclin that interacts with two members of the CDC2 gene family. Cell 66: 1217-1228, 1991.

24. Tirone F: The gene PC3(TIS21/BTG2), prototype member of the PC3/BTG/TOB family: regulator in control of cell growth, differentiation, and DNA repair? J Cell Physiol 187: 155-165, 2001.

25. Nicholson DW and Thornberry NA: Apoptosis. Life and death decisions. Science 299: 214-215, 2003.

26. Corjay MH, Kearney MA, Munzer DA, Diamond SM and Stoltenborg JK: Antiproliferative gene BTG1 is highly expressed in apoptotic cells in macrophage-rich areas of advanced lesions in Watanabe heritable hyperlipidemic rabbit and human. Lab Invest 78: 847-858, 1998.

27. Lee H, Cha S, Lee MS, Cho GJ, Choi WS and Suk K: Role of antiproliferative B cell translocation gene-1 as an apoptotic sensitizer in activation-induced cell death of brain microglia. J Immunol 171: 5802-5811, 2003.

28. Nahta R, Yuan LX, Fiterman DJ, Zhang L, Symmans WF, Ueno NT and Esteva FJ: B cell translocation gene 1 contributes to antisense Bcl-2-mediated apoptosis in breast cancer cells. Mol Cancer Ther 5: 1593-1601, 2006.

29. Wiseman BS and Werb Z: Stromal effects on mammary gland development and breast cancer. Science 296: 1046-1049, 2002.

30. Alok C and Bharat B: Nuclear factor-kappa Band cancer: its role in prevention and therapy. Biochem Phamacol 64: 883-888, 2002.

31. Virós D, Camacho M, Zarraonandia I, García J, Quer M, Vila L and León X: Prognostic role of MMP-9 expression in head and neck carcinoma patients treated with radiotherapy orchemoradiotherapy. Oral Oncol 49: 322-325, 2013. 